\title{
Value of Pre- and Post-Treatment Platelet Indices in Patients with Immune Thrombocytopenic Purpura
}

\author{
Iman Mohamed Omar, Soha Abuelela, Noha Emam \\ Clinical Pathology Department, Faculty of Medicine, Ain Shams University, Cairo, Egypt \\ Email:soha_abuelela@med.asu.edu.eg
}

How to cite this paper: Omar, I.M., Abuelela, S. and Emam, N. (2018) Value of Pre- and Post-Treatment Platelet Indices in Patients with Immune Thrombocytopenic Purpura. Journal of Biosciences and Medicines, 6, 11-24. https://doi.org/10.4236/jbm.2018.69002

Received: July 24, 2018

Accepted: September 8, 2018

Published: September 11, 2018

Copyright $(9) 2018$ by authors and Scientific Research Publishing Inc. This work is licensed under the Creative Commons Attribution International License (CC BY 4.0).

http://creativecommons.org/licenses/by/4.0/

\section{(c) () Open Access}

\begin{abstract}
Introduction: Immune thrombocytopenic purpura (ITP) is an acquired disorder characterized by isolated thrombocytopenia. There is no "gold standard" test that can establish the diagnosis of primary ITP. Therefore, the diagnosis is one of exclusion and is based on patient history, physical examination, complete blood count, and blood smear review. Platelet indices are biomarkers of platelet activation, allowing extensive investigations focusing on the diagnostic and prognostic values in various diseases without extra-costs. Mean platelet volume (MPV), platelet distribution width (PDW) and plateletcrit (PCT) were investigated aiming at evaluating the correlation between pre- and post-treatment values in ITP patients. The sensitivity and specificity of platelet indices for assessment of their predictive value for treatment response were also evaluated. Methods: Fifty newly diagnosed primary ITP patients were selected from the Hematology Clinics of Ain Shams University Hospitals and classified into pediatric and adult groups. Platelet count, MPV, PDW, PCT have been recorded for all pre and post-treatment CBC samples using Beckman Coulter LH750 analyzer. Results: In pediatrics post-treatment MPV and PDW were significantly decreased while PCT was increased when compared to their values at the time of presentation $(\mathrm{P}<$ 0.01). The same results were obtained in adult patients, except for PDW which did not attain any significance. A significant correlation was found between pre-treatment values of these platelet indices and treatment response in pediatrics, while no significance was found between pre-treatment PDW and treatment response in adults. Conclusion: Increased pre-treatment MPV and PDW, and decreased PCT can provide diagnostic and predictive value for treatment response in ITP patients.
\end{abstract}

\section{Keywords}

ITP, Treatment, Response, Platelet Indices, Mean Platelet Volume, 
Platelet Distribution Width, Plateletcrit

\section{Introduction}

Immune thrombocytopenic purpura (ITP) is an immune-mediated acquired bleeding disorder of adults and children described by transient or persistent reduction of the platelet number and, depending on the level of thrombocytopenia, increased risk of bleeding. A platelet count under $100 \times 10^{9} / \mathrm{L}$ was established as the threshold for diagnosis [1]. Increased platelet destruction is clearly assumed to have the key role in the pathogenesis of ITP; however impaired platelet production is likewise important in many cases. In adults, around $40 \%$ of ITP cases may have decreased platelet renewal, mirroring the inhibitory effect of platelet autoantibodies on megakaryopoiesis [2].

ITP in children is generally an acute self-limiting problem with severe clinical presentation; they regularly present with sudden onset of petechiae or purpura few days or weeks after an infectious illness. But in adults it has a tendency to be a chronic disease with insidious onset and persistent course lasting for many years with recurrent relapses. ITP is classified as primary or as secondary to an underlying disorder, and as acute (of six months or less in duration) or chronic (when symptoms last over 6 months) [3].

Infection with hepatitis $B$, hepatitis $C$, human immunodeficiency virus (HIV), Helicobacter pylori, and coexistence of systemic lupus erythematosus (SLE), antiphospholipid syndrome (APS), or common variable immunodeficiency (CVID) are potential fundamental etiology to secondary ITP, and these are recognized in approximately $20 \%$ of ITP patients. The diagnosis of ITP is based basically on the history, physical examination, complete blood count ( $\mathrm{CBC}$ ), and examination of the peripheral smear, which should exclude other causes of thrombocytopenia assuming that they are compatible with the diagnosis of ITP and exclude atypical findings that are uncommon in ITP or suggest different etiologies [4].

On CBC, isolated thrombocytopenia is the hallmark of ITP. The guidelines of the American Society of Hematology stated that a bone marrow examination is unnecessary in patients with the typical features of ITP regardless the age of the patient [5]. Owing to the fact that BM examination is an invasive and discomforting procedure, and is unsuitable for frequent follow-up in thrombocytopenic patients; hence a sensitive and non-invasive test, capable of evaluating the thrombopoietic activity, would be of substantial clinical value. Management of patients with ITP relies upon corticosteroids or intra-venous immunoglobulin (IVIG) as initial first-line treatment. In the presence of low platelet counts with active bleeding or the patients fail to respond to first line drug, immunosuppressive agents, as second-line drugs (e.g. azathioprine or cyclophosphamide) are used. Treatment is generally started at platelet counts under $20 \times 10^{9} / \mathrm{L}$, as spon- 
taneous intra-cerebral hemorrhage is a major hazard [6].

Platelet indices as mean platelet volume (MPV), platelet distribution width (PDW), and plateletcrit (PCT) are available on automated hematology analyzers, being derived from the platelet distribution curve, and easily addressed along with the platelet count. They are sensitive, non-invasive, inexpensive biomarkers and can help in diagnosis and prognosis of many diseases [7]. MPV is a marker of platelet size, function, and activation, as large platelets contain more dense granules and produce more thromboxane A2 than smaller ones. Increasing the number of megakaryocytes and supporting the diagnosis of ITP is associated with larger MPV value [8]. PDW (coefficient of PLT variation) directly measures variability in platelet size, changes with platelet activation, and reflects the heterogeneity in platelet morphology [9]. Under physiological conditions, there is a direct relationship between MPV and PDW; both usually change in the same direction. An increase in both MPV and PDW occurs due to platelet activation, in which platelet swelling and pseudopodia formation occur as large platelets are metabolically and enzymatically more active than small platelets [10].

PCT is another platelet parameter, which is a reliable measurement of platelet biomass. It is the volume occupied by platelets in the blood as a percentage and is calculated using MPV value and platelet count [11]. Although Platelet indices are useful markers, there are several technical issues limiting their clinical use. The main influential factors are: the type of hematology analyzer, the anticoagulant applied, and the time from sampling to analysis [12]. EDTA causes gradual platelet swelling, thus increase in MPV during the first 6 hours when measured by impedance method and decreases by $10 \%$ when determined by an optical method. Time delay probably affects PDW also [13]. MPV can be measured accurately by both methods of anticoagulation; EDTA and citrate if analysis is performed within $1 \mathrm{~h}$ of sampling, however; it's known that citrate is the best anticoagulant in managing pseudo-thrombocytopenia but it lowers MPV value [14].

As mentioned in the literature, platelet indices allow extensive clinical investigations focusing on the diagnostic and prognostic values in a variety of settings without bringing extra costs. This study was conducted with the objectives of analyzing the correlation between pre- and post-treatment platelet indices in ITP patients and evaluating the sensitivity and specificity of platelet indices as a predictor of treatment response.

\section{Materials and Methods}

\subsection{Materials}

This study was conducted on fifty newly diagnosed patients with primary ITP selected from the Hematology Clinics of Ain Shams University Hospitals. Their diagnosis was established according to new consensus of terminology and definitions where platelet count is $<100 \times 10^{9} / \mathrm{L}$, the duration $<3$ months from diagnosis and after exclusion of all causes of thrombocytopenia [5]. All patients 
were subjected to complete history taking focusing on recent fever or viral illness, concurrent drug intake and bleeding history. Patients were divided into 2 groups a pediatric patient group which included 25 children, 14 females and 11 males and an adult group. The children's age ranged from 2 months to 12 years. Their bone marrow examination showed a mildly hypercellular specimen in almost most of the patients with moderately increased megakaryopoiesis. Corticosteroids were given as first line treatment in 18 patients while IVIG was given to 7 patients from the start because of the presence of significant bleeding in those patients.

The adult patients group included 21 females and 4 males. Their age ranged from 18 to 42 years. Their bone marrow examination ranged from normocellular to mildly hypercellular specimens with increased megakaryopoiesis. Corticosteroids were the first line of treatment for all patients. Patients with ITP secondary to medications, viral infections, autoimmune conditions or leukemia were excluded from the study.

The duration of treatment for all studied patients didn't exceed 4 weeks duration.

\subsection{Methods}

Two milliliters (ml) venous blood were collected from each patient under complete aseptic conditions in vacutainer tubes containing $1.5 \pm 0.25 \mathrm{mg}$ dipotassium EDTA per $1 \mathrm{ml}$ blood and analysis was done within 2 hours from collection. The following parameters have been recorded for all the studied samples: platelet count, MPV, PDW, PCT using Beckman Coulter LH750 hematology analyzer (Beckman Coulter Inc., USA) that utilizes impedance resistance technology for platelet counting. Platelet counts had been also confirmed by the examination of an adequately prepared leishman-stained peripheral blood smear by optical microscopy to exclude pseudo-thrombocytopenia.

Two samples were collected from all patients:

- One at the time of diagnosis and before the start of treatment (pre-treatment $\mathrm{CBC})$.

- The second sample was collected within $(7-28)$ days after the treatment (post treatment $\mathrm{CBC}$ ).

Patients were divided according to the response to treatment into:

- A complete response was defined as platelet counts $>100 \times 10^{9} / \mathrm{L}$.

- A partial response was defined as platelet counts $30-100 \times 10^{9} / \mathrm{L}$ and platelet counts have reached at least 2 folds from baseline.

- No response was defined as platelet counts $<30 \times 10^{9} / \mathrm{L}$ or platelet counts have not reached at least 2 folds from baseline [5].

\subsection{Statistical Analysis}

The collected data was revised, coded, tabulated and introduced to a PC using Statistical package for Social Science (SPSS 15.0.1 for windows; SPSS Inc., Chi- 
cago, IL, 2001). Mean, Standard deviation ( \pm SD) and range were used for parametric numerical data, while Median and Interquartile range (IQR) were used for non-parametric data. Student $\mathrm{T}$ Test was used to assess the statistical significance of the difference between two study group means. Mann Whitney Test ( $U$ test) was used for the difference of a non-parametric variable between two groups. ANOVA test was used for the difference between more than two group means. A P-value of $<0.05$ was considered statistically significant.

\section{Results}

Out of 25 pediatric patients, 11 (44\%) presented with petechiae and $5(20 \%)$ had ecchymosis. On examination, splenomegaly was observed in 5 (20\%) of patients and hepatomegaly was detected in only $2(8 \%)$ patients. Out of 25 adult patients, vaginal bleeding was the main presenting symptom in $8(32 \%)$ of patients. On examination, splenomegaly was observed in 3 (12\%), while 2 (8\%) had hepatomegaly.

In the pediatric group, the pre-treatment platelet counts ranged from $7-18 \times$ $10^{9} / \mathrm{L}$ (median 10), while the post-treatment counts ranged from $65-117 \times 10^{9} / \mathrm{L}$ (median 98). The pre-treatment platelet counts in adult group ranged from 7 $18 \times 10^{9} / \mathrm{L}$ (median 12), while the post-treatment counts ranged from $63-144 \times$ $10^{9} / \mathrm{L}$ (median 128). Pre and post treatment platelet indices values are given in Table 1.

Patients were grouped according to the response to treatment into complete response group which included $44 \%$ of pediatric patients and $60 \%$ of adults and partial response to treatment to which $26 \%$ of children and $28 \%$ of adult patients were assigned. Only 5 (20\%) of children and $3(12 \%)$ of adults did not respond to the given treatment.

Neither pre-treatment platelet counts and indices nor post-treatment values

Table 1. Pre and Post treatment platelet indices of studied patients.

\begin{tabular}{|c|c|c|c|}
\hline & \multirow{3}{*}{ Parameter } & Pediatrics & Adults \\
\hline & & Range & Range \\
\hline & & Mean \pm SD & Mean \pm SD \\
\hline \multirow{6}{*}{ 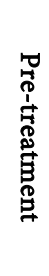 } & \multirow{2}{*}{ MPV (fl) } & $8.6-12.6$ & $8-12$ \\
\hline & & $10.6 \pm 2$ & $10 \pm 2$ \\
\hline & \multirow{2}{*}{ PDW (\%) } & $15-19$ & $13-18$ \\
\hline & & $16.9 \pm 2.4$ & $15.7 \pm 2.7$ \\
\hline & \multirow{2}{*}{ PCT (\%) } & $0-0.02$ & $0-0.04$ \\
\hline & & $0.01 \pm 0.01$ & $0.02 \pm 0.02$ \\
\hline \multirow{6}{*}{ 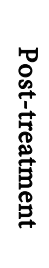 } & \multirow{2}{*}{ MPV (fl) } & $8-11$ & $7.7-10.3$ \\
\hline & & $9.5 \pm 1.5$ & $9 \pm 1.3$ \\
\hline & \multirow{2}{*}{ PDW (\%) } & $14-18$ & $13.3-17.2$ \\
\hline & & $16 \pm 2$ & $15.5 \pm 1.7$ \\
\hline & \multirow{2}{*}{ PCT (\%) } & $0.02-0.1$ & $0-0.2$ \\
\hline & & $0.07 \pm 0.05$ & $0.1 \pm 0.1$ \\
\hline
\end{tabular}


or response to treatment showed any statistically significant difference $(\mathrm{P}>0.05)$ between adult and pediatric patients groups, except for post-treatment platelet count and PCT which revealed a higher statistically significant difference $(\mathrm{P}<$ 0.05 ) between both groups (Table 2).

We found that the pre-treatment values for MPV were high in both patient groups, and that MPV was normalized after first line treatment and the comparison attained a highly statistically significant difference $(\mathrm{P}<0.01)$. The same highly significant difference was detected between the pre and post-treatment values for PCT in both groups; in the same direction with platelet counts; which were higher after initiation of first line treatment. We could not obtain significant statistical differences between PDW values $(\mathrm{P}>0.05)$ at the time of diagnosis and after first line treatment in adults; however, the comparison yielded a statistically significant difference $(P<0.05)$ in children.

In an attempt to further study the usefulness of platelet indices as predictors for treatment response in acute ITP patients, the correlation between the pre-treatment platelet indices and treatment response among the studied groups was evaluated. A highly statistically significant difference was detected between patients with complete response and non-responders regarding all platelet indices in both patient groups (Figure 1). Besides, a highly significant difference regarding MPV and PDW was detected between patients who achieved partial response and non-responders, yet there wasn't any significant difference $(\mathrm{P}>$ 0.05) regarding platelet count and PCT in children; while in adults, neither of the studied platelet indices reached a statistically significant difference $(P>0.05)$

Table 2. Comparison between adults and pediatric patients regarding pre- and post-treatment platelet count and indices and treatment response.

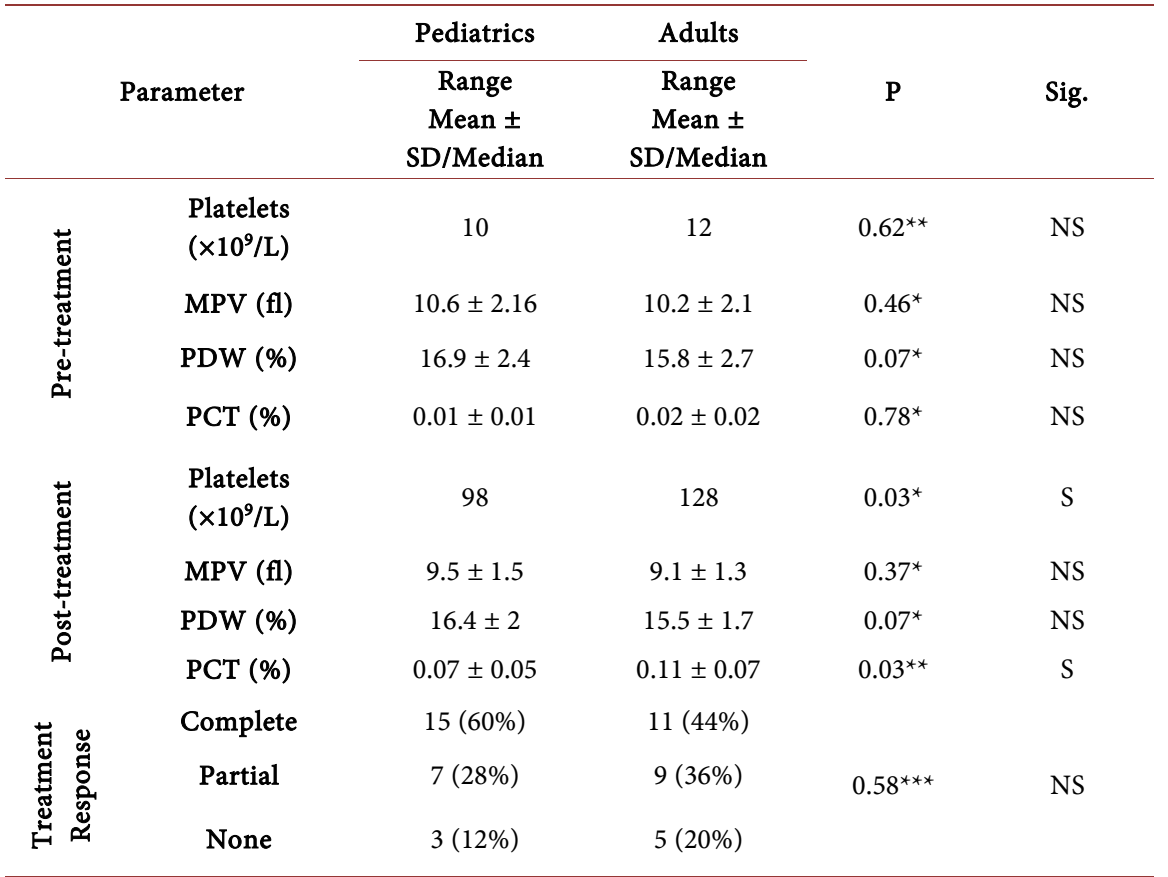

${ }^{\star}$ Student T test, ${ }^{*}$ Mann-Whitney Test, ${ }^{* *}$ Chi square test, NS: non-significant, S: significant. 


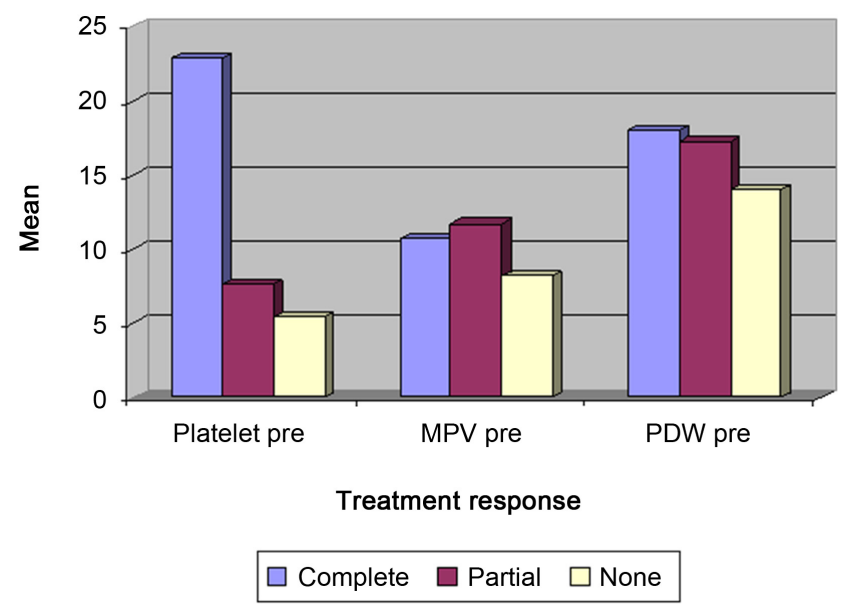

Figure 1. Pre-treatment platelet indices and treatment response among children.

\section{(Figure 2).}

Accordingly, we tried to settle cut off values for platelet indices that can predict the patients' response to treatment using the ROC curve. The best cut off level for MPV was $>8.4 \mathrm{fL}$, with a diagnostic sensitivity $92.8 \%$ and specificity $87.5 \%$. The negative predictive value was $70 \%$ and positive predictive value was $97.5 \%$. While the best cut off level for PDW was $>15 \%$, with a diagnostic sensitivity $78.5 \%$ and specificity $87.5 \%$, the negative predictive value was $43.8 \%$ and positive predictive value was $97.1 \%$. As for PCT, a cut off value of $>0.01$, showed a diagnostic sensitivity $57 \%$ and specificity $100 \%$. The negative predictive value was $30.8 \%$ and positive predictive value was $100 \%$ (Table 3 ).

\section{Discussion}

Our study was conducted on fifty newly diagnosed patients with primary ITP, in order to study MPV, PDW and PCT values at the time of diagnosis and after the start of first line treatment. The aim was to demonstrate the changes of platelet indices in response to the given treatment and to assess their predictive value at the time of presentation for the response to treatment.

In 2006, Segal and Powe suggested that the incidence in adults is approximately equal for both sexes except in the mid-adults (3rd - 6th decades), when the disease is more prevalent in women [15]. In our study, $84 \%$ of adult patients were females with male to female ratio 1:5.25. This comes in agreement with Libert et al., 2010 who suggested that autoimmune disorders are associated with female gender; it may be an explanation for the greater number of adult women with ITP [16]. In the pediatric group, $60 \%$ of patients were females with male to female ratio 1:1.5. Malpass and colleagues, 2015 also found that out of total 42 pediatric patients with ITP, twenty-seven (64\%) of them were females [17]. On the contrary, a prospective registry has documented a higher rate of acute ITP in males than females (54.8\% vs $45.2 \%$ ) [18]. This contradiction is also found with studies of Kühne and colleagues (2003) and Shirahata et al., 2009 who also 


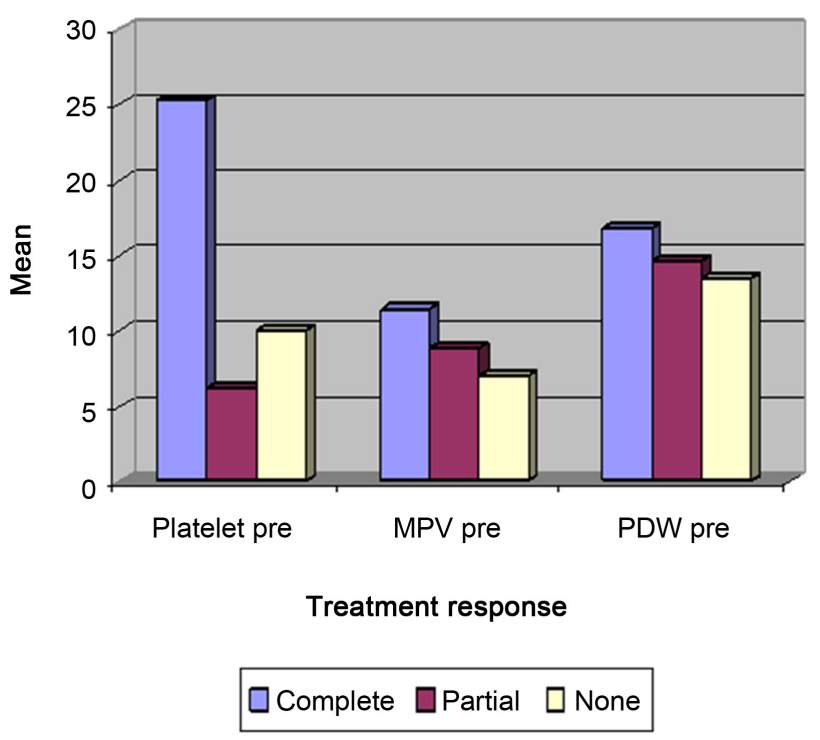

Figure 2. Pre-treatment platelet indices and treatment response among adults.

Table 3. ROC curve analysis for pre-treatment platelet indices.

\begin{tabular}{lcccccc}
\hline Cutoff level & AUC $(\mathrm{CI})$ & Sensitivity & Specificity & PPV & NPV & P (sig.) \\
\hline MPV > 8.4 & $0.857(0.729$ to 0.940$)$ & 92.86 & 87.50 & 97.5 & 70.0 & $0.001(\mathrm{HS})$ \\
PDW > 15 & $0.871(0.745$ to 0.949$)$ & 78.57 & 87.50 & 97.1 & 43.8 & $0.001(\mathrm{HS})$ \\
PCT $>\mathbf{0 . 0 1}$ & $0.856(0.727$ to 0.939$)$ & 57.14 & 100.00 & 100.0 & 30.8 & $0.001(\mathrm{HS})$ \\
\hline
\end{tabular}

demonstrated that male gender dominates among pediatric patients [19] [20]. They added that reasons for their observed high proportion of males in childhood ITP remain unknown.

Regarding signs and symptoms, Neylon et al., 2003 added that petechiae, ecchymosis, epistaxis, gum bleeding and genital tract bleeding are the most common initial manifestations of ITP [21]. In our study groups, petechiae were the most common presenting symptom (44\%) in children, while in adults; vaginal bleeding was the most common accounting for $32 \%$ of the cases as 21 out of 25 (84\%) patients were females. Mild splenomegaly may be found in younger patients, but moderate or massive splenomegaly suggests an alternative cause. In our study, mild splenomegaly was found in $20 \%$ of pediatric patients which is more than expected, challenging the diagnosis of primary ITP. This can be attributed, in part to occurrence of ITP in children usually following an apparent viral infection, and in another to the presence of lax abdomen or thinner abdominal wall in children. Similarly, George et al., 1996 suggested that the spleen may be palpable in $12 \%$ of children with ITP [22].

In the current study, the pre-treatment mean MPV values were increased in the studied groups relative to the reference range. These increased values in ITP have been recognized already since 1983 by Van der Lelie [23]. Likely, korkomaz and his colleagues 2013, and Adly and coworkers, 2014 found that MPV shows 
higher levels in ITP patients than in other causes of thrombocytopenia [24] [25]. Therefore in agreement with Kaito et al., 2005 and Ntaios et al., 2008 we concluded that increased MPV may provide a reliable positive diagnosis of ITP in case of thrombocytopenic patients [26] [27]. This can be explained by the findings of Budak and colleagues in 2016 who revealed that when platelet production is decreased, young platelets become bigger and more active, and MPV levels increase, also during activation, platelets' shapes change from biconcave discs to spherical with pseudopods formation [28].

On the contrary, Ahmed et al., 2004 and Donato et al., 2009 found that MPV at diagnosis of ITP was lower than the normal control subjects [29] [30]. As regards PDW, pre-treatment PDW values in pediatric and adult groups in our study are considered high relatively to the reference range. This can be explained by Zhang et al., 2014 who added that both newly produced PLTs with large volume and mature PLTs with small volume are simultaneously present in the blood; therefore, both MPV and PDW will be increased correspondingly [31]. Moreover, Vagdatli and colleagues, 2010 stated that under physiological conditions, there is a direct relationship between MPV and PDW; both usually change in the same direction [10]. As for PCT, low pre-treatment PCT in children and in adult is also found in the results of the study by korkomaz and his colleagues 2013 [24]. In 2005, Kaito and colleagues studied the platelet indices as a diagnostic marker in ITP and found that MPV and PDW were significantly higher in ITP than in other causes of thrombocytopenia, and the sensitivity and specificity of platelet indices were sufficient to enable a diagnosis of ITP [26]. In our study, MPV and PDW values were significantly high and PCT was significantly low, this comes in agreement with other studies by Ntaios et al., 2008 and Negash $M$ and Tsegaye, 2016, all independently predicted a favorable outcome of acute ITP [27] [32].

In 2011, Seth stated that initial treatment of newly diagnosed patients for the first time is aimed at rapidly obtaining a safe platelet count $\left(>20-30 \times 10^{9} / \mathrm{L}\right)$ in order to prevent or stop hemorrhages and to ensure an acceptable quality of life with minimal treatment related toxicity [33]. In primary ITP, corticosteroids are the standard initial therapeutic agents. It is given orally at a dose of $0.5-2 \mathrm{mg} / \mathrm{kg}$ daily, when corticosteroids used alone, $65 \%-70 \%$ of patients respond. In our study, we obtained high response rate (complete response + partial response) in $42(84 \%)$ of the studied patients to the initial treatment, while $8(16 \%)$ patients did not achieve a satisfactory response. Following the treatment, the post-treatment platelet counts increased in both studied groups, while that of MPV decreased following the start of treatment. This decrease in the post-treatment values may be attributed to achieving response in most of our studied patients to the first line treatment. Similar results were obtained regarding PDW values at the time of presentation and following treatment in both groups.

This is consistent with the results obtained by korkomaz and his colleagues 
(2013), in which MPV was found to be higher in patients diagnosed with ITP, and it normalized after first-line treatment [24]. An explanation for this finding may be how steroids therapy improves platelet survival but doesn't increase platelet production (platelet turnover) by significantly decreasing antibody production and suppressing RES phagocytic function. Subsequently, following the start of steroids, an increase in the small mature platelets and decrease in the activated large platelets occur [34] [35]. In both pediatrics and adults, PCT was significantly higher after the start of treatment, compared to its values at the time of diagnosis. This change in PCT following the administration of the first line treatment was consistent with the change of PLT count as proved by Liu and colleagues, 2016 and Korkmaz et al., 2013 who found increased PCT following the treatment [36] [24]. Following treatment, PLT count and PCT gradually increased, whereas PDW and MPV gradually decreased. PLT count was negatively related to MPV, while MPV was positively related to PDW [36]. These studies were also consistent with our results and with those of Fan and Wei (2014) which suggested that the change of platelet parameters was beneficial to the diagnosis and efficacy interpretation of ITP patients [37].

In our study comparing the studied platelet indices before and after treatment, it revealed that a high statistically significant difference was obtained between MPV and PCT values at the time of diagnosis and after the start of first line treatment in both groups $(\mathrm{P}<0.01)$ and PDW showed only statistically significant difference in pediatrics $(\mathrm{P}<0.05)$, while no significance was elicited in adults $(\mathrm{P}>0.05)$. Similarly, Korkmaz and co-workers, 2013 reported that MPV and PCT values show a high statistically significant difference between the time of diagnosis and after the start of first line treatment $(\mathrm{P}<0.01)$ but PDW didn't show any statistically significant difference [24]. In the contrary, Kim et al., in 2002 found that MPV shows statistically significant difference between the time of diagnosis and after the treatment but with increase in its values, while PDW showed no significance [38]. This deviation in results could be attributed to the difference in the selection of the patients where Kim et al., (2002) selected his patients from children only, the initial treatment given was IVIG as first line and they used Coulter STKS as the hemtology analyzer [38]. Korkmaz et al., 2013 study was based on 81 patients and used steroids and IVIG as initial therapy and their analyzer was Siemens Advia 2120 [24]. As mentioned the difference in the type of the hematology analyzer used could affect the results, as older automated analyzers, used in these studies, cannot discriminate platelets from other similar-sized particles such as fragmented red or white cells, cell debris and immune complexes. Moreover, they do not count large or giant platelets because they cannot be differentiated from red cells. Furthermore, many studies in the literature have shown that MPV is dependent on number of variables, including time of analysis after venipuncture, anticoagulant used, specimen storage temperature and cell counter technologies [36] [39].

As shown in our results, all platelet indices values at the time of diagnosis of acute ITP in both adults and children showed a highly statistically significant 
difference between patients who achieved complete response to first line treatment and non-responders. Moreover, pre-treatment MPV and PDW levels were significantly different in patients who achieved partial response from non-responders. Hence, we assumed that pre-treatment platelet indices values could help predict how acute ITP patients will respond to first line treatment; either by achieving complete or partial response or not responding at all, thus detecting failure of first line treatment in those patients earlier and starting them on second line drugs before occurrence of bleeding due to persistently low platelet counts. Accordingly, we tried to settle cut off values to the platelet indices that can predict the patients' response to treatment using the ROC curve. Currently, physicians tend to focus on the relationship between the change of platelet count only and diseases. Simultaneous measurement of all the platelet indices will provide us a valid tool for measuring disease severity and an insight into the potential etiology that resulted in platelets' indices changes. The findings of the current study as well as other studies have demonstrated that the use of platelet parameters is convenient, reliable and valuable to the diagnosis and follow up in ITP patients as it avoids the pain from bone marrow biopsy [36].

\section{Conflicts of Interest}

The authors declare no conflicts of interest regarding the publication of this paper.

\section{References}

[1] Rodeghiero, F., Stasi, R., Gernsheimer, T., Michel, M., Provan, D., Arnold, D.M., Bussel, J.B., Cines, D.B., Chong, B.H., Cooper, N., Godeau, B., Lechner, K., Mazzucconi, M.G., McMillan, R., Sanz, M.A., Imbach, P., Blanchette, V., Kühne, T., Ruggeri, M. and George (2009) Standardization of Terminology, Definitions and Outcome Criteria in Immune Thrombocytopenic Purpura of Adults and Children: Report from an International Working Group. Blood, 113, 2386-2393. https://doi.org/10.1182/blood-2008-07-162503

[2] Blanchette, V. and Bolton-Maggs, P. (2008) Childhood Immune Thrombocytopenic Purpura: Diagnosis and Management. Pediatric Clinics of North America, 55, 393-420. https://doi.org/10.1016/j.pcl.2008.01.009

[3] Gernsheimer, T. (2008) Epidemiology and Pathophysiology of Immune Thrombocytopenic Purpura. European Journal of Haematology, 80, 3-8. https://doi.org/10.1111/j.1600-0609.2007.00998.x

[4] Cines, D.B., Bussel, J.B., Liebman, H.A. and LuningPrak, E.T. (2009) The ITP Syndrome: Pathogenic and Clinical Diversity. Blood, 113, 6511-6521. https://doi.org/10.1182/blood-2009-01-129155

[5] Neunert, C., Lim, W., Crowther, M., Cohen, A., Solberg Jr., L. and Crowther, M.A. (2011) The American Society of Hematology 2011 Evidence-Based Practice Guideline for Immune Thrombocytopenia. Blood, 117, 4190-4207. https://doi.org/10.1182/blood-2010-08-302984

[6] Vianelli, N., Valdre, L. and Fiacchini, M. (2001) Long-Term Follow-Up of Idiopathic Thrombocytopenic Purpura in 310 Patients. Haematologica, 86, 504-509.

[7] Gao, Y., Li, Y., Yu, X., Guo, S., Ji, X., Sun, T., Lan, C., Lavergne, V. and Ghannoum, 
L. (2014) The Impact of Various Platelet Indices as Prognostic Markers of Septic Shock. PloS ONE, 9, e103761. https://doi.org/10.1371/journal.pone.0103761

[8] Rajantie, J., Javela, K., Joutsi-Korhonen, L. and Kekomäki, R. (2004) Chronic Thrombocytopenia of Childhood: Use of Non-Invasive Methods in Clinical Evaluation. European Journal of Haematology, 72, 268-272.

https://doi.org/10.1111/j.1600-0609.2004.00215.x

[9] Wiwanitkit, V. (2004) Plateletcrit, Mean Platelet Volume, Platelet Distribution Width: Its Expected Values and Correlation with Parallel Red Blood Cell Parameters. Clinical and Applied Thrombosis/ Hemostasis, 10, 175-178. https://doi.org/10.1177/107602960401000208

[10] Vagdatli, E., Gounari, E., Lazaridou, E., Katsibourlia, E., Tsikopoulou, F. and Labrianou, I. (2010) Platelet Distribution Width: A Simple, Practical and Specific Marker of Activation of Coagulation. Hippokratia, 14, 28-32.

[11] Akpinar, I., Sayin, M.R., Gursoy, Y.C., Aktop, Z., Karabag, T., Kucuk, E., Sen, N., Aydin, M., Kiran, S., Buyukuysal, M.C. and Haznedaroglu, I.C. (2014) Plateletcrit and Red Cell Distribution Width Are Independent Predictors of the Slow Coronary Flow Phenomenon. Journal of Cardiology, 63, 112-118. https://doi.org/10.1016/j.jjcc.2013.07.010

[12] Vinholt, P.J., Hvas, A.M. and Nybo, M. (2014) An Overview of Platelet Indices and Methods for Evaluating Platelet Function in Thrombocytopenic Patients. European Journal of Haematology, 92, 367-376. https://doi.org/10.1111/ejh.12262

[13] Chandra, H., Chandra, S., Rawat, A. and Verma, S.K. (2010) Role of Mean Platelet Volume as Discriminating Guide for Bone Marrow Disease in Patients with Thrombocytopenia. International Journal of Laboratory Hematology, 32, 498-505. https://doi.org/10.1111/j.1751-553X.2009.01212.x

[14] Dastjerdi, M.S., Emami, T., Najafian, A. and Amini, M. (2006) Mean Platelet Volume Measurement, EDTA or Citrate? Hematology, 11, 317-319. https://doi.org/10.1080/10245330600954163

[15] Segal, J.B. and Powe, N.R. (2006) Prevalence of Immune Thrombocytopenia: Analyses of Administrative Data. Journal of Thrombosis and Haemostasis, 4, 2377-2383. https://doi.org/10.1111/j.1538-7836.2006.02147.x

[16] Libert, C., Dejager, L. and Pinheiro, I. (2010) The X Chromosome in Immune Functions: When a Chromosome Makes the Difference. Nature Reviews Immunology, 10, 594-604. https://doi.org/10.1038/nri2815

[17] Malpass, J., Lavigne, J.L., Pope, J. and Oesterheld, J. (2015) Investigation of Mean Platelet Volume as a Predictor of Acute versus Chronic Immune Thrombocytopenic Purpura in Children: An Exploratory Analysis. Clinical Nursing Studies, 3, 23.

[18] Arceci, R.J., Hann, I.M., Smith, O.P. and Hoffbrand, A.V. (2006) Pediatric Hematology. Blackwell Pub., Malden. https://doi.org/10.1002/9780470987001

[19] Kühne, T., Buchanan, G.R., Zimmerman, S., Michaels, L.A., Kohan, R., Berchtold, W. and Imbach, P. (2003) A Prospective Comparative Study of 2540 Infants and Children with Newly Diagnosed Idiopathic Thrombocytopenic Purpura (ITP) from the Intercontinental Childhood ITP Study Group. Journal of Pediatrics, 143, 605-608. https://doi.org/10.1067/S0022-3476(03)00535-3

[20] Shirahata, A., Fujisawa, K. and Ishii, E. (2009) A Nationwide Survey of Newly Diagnosed Childhood Idiopathic Thrombocytopenic Purpura in Japan. Journal of Pediatric Hematology/ Oncology, 31, 27-32. https://doi.org/10.1097/MPH.0b013e318190d44e

[21] Neylon, A.J., Saunders, P.W., Howard, M.R., Proctor, S.J. and Taylor, P.R. (2003) 
Clinically Significant Newly Presenting Autoimmune Thrombocytopenic Purpura in Adults: A Prospective Study of a Population-Based Cohort of 245 Patients. British Journal of Haematology, 122, 966-974. https://doi.org/10.1046/j.1365-2141.2003.04547.x

[22] George, J.N., Woolf, S.H., Raskob, G.E., Wasser, J.S., Aledort, L.M., Ballem, P.J., Blanchette, V.S., Bussel, J.B., Cines, D.B., Kelton, J.G. and Lichtin, A.E. (1996) Idiopathic Thrombocytopenic Purpura: A Practice Guideline Developed by Explicit Methods for the American Society of Hematology. Blood, 88, 3-40.

[23] Van der Lelie, J. and Von dem Borne, A.K. (1982) Increased Mean Platelet Volume in Septicaemia. Journal of Clinical Pathology, 36, 693-696. https://doi.org/10.1136/jcp.36.6.693

[24] Korkmaz, S., Uslu, A.U., Aydin, B., Dogan, O. and Sencan, M. (2013) Pre-Treatment and Post-Treatment Changes in Platelet Indices in Patients with Immune Thrombocytopenia. Saudi Medical Journal, 34, 591-596.

[25] Adly, A.A. (2014) Pathophysiology of Immune Thrombocytopenic Purpura: A Bird's-Eye View. Egyptian Journal of Pediatric Allergy and Immunology, 12, 49-61.

[26] Kaito, K., Otsubo, H., Usui, N., Yoshida, M., Tanno, J., Kurihara, E., Matsumoto, K., Hirata, R., Domitsu, K. and Kobayashi, M. (2005) Platelet Size Deviation Width, Platelet Large Cell Ratio, and Mean Platelet Volume Have Sufficient Sensitivity and Specificity in the Diagnosis of Immune Thrombocytopenia. British Journal of Haematology, 128, 698-702. https://doi.org/10.1111/j.1365-2141.2004.05357.x

[27] Ntaios, G., Papadopoulos, A., Chatzinikolaou, A., Saouli, Z., Karalazou, P., Kaiafa, G., Girtovitis, F., Kontoninas, Z., Savopoulos, C., Hatzitolios, A. and Alexiou-Daniel, S. (2008) Increased Values of Mean Platelet Volume and Platelet Size Deviation Width May Provide a Safe Positive Diagnosis of Idiopathic Thrombocytopenic Purpura. Acta Haematologica, 119, 173-177. https://doi.org/10.1159/000135658

[28] Budak, Y.U., Polat, M. and Huysal, K. (2016) The Use of Platelet Indices, Plateletcrit, Mean Platelet Volume and Platelet Distribution Width in Emergency Non-Traumatic Abdominal Surgery: A Systematic Review. Biochemiamedica, 26, 178-193. https://doi.org/10.11613/BM.2016.020

[29] Ahmed, S., Siddiqui, A.K., Shahid, R.K., Kimpo, M., Sison, C.P. and Hoffman, M.A. (2004) Prognostic Variables in Newly Diagnosed Childhood Immune Thrombocytopenia. American Journal of Hematology, 77, 358-362.

https://doi.org/10.1002/ajh.20205

[30] Donato, H., Picón, A., Martinez, M., Rapetti, M.C., Rosso, A., Gomez, S., Rossi, N., Bacciedoni, V., Schvartzman, G., Riccheri, C. and Costa, A. (2009) Demographic Data, Natural History, and Prognostic Factors of Idiopathic Thrombocytopenic Purpura in Children: A Multicentered Study from Argentina. Pediatric Blood \& Cancer, 52, 491-496. https://doi.org/10.1002/pbc.21872

[31] Zhang, Z., Xu, X., Ni, H. and Deng, H. (2014) Platelet Indices Are Novel Predictors of Hospital Mortality in Intensive Care Unit Patients. Journal of Critical Care, 29, 885. https://doi.org/10.1016/j.jcrc.2013.09.002

[32] Negash, M. and Tsegaye, A. (2016) Diagnostic Predictive Value of Platelet Indices for Discriminating Hypo Productive versus Immune Thrombocytopenia Purpura in Patients Attending a Tertiary Care Teaching Hospital in Addis Ababa, Ethiopia. BMC Hematology, 16, 18. https://doi.org/10.1186/s12878-016-0057-5

[33] Seth, T. (2011) Current Management of Idiopathic Thrombocytopenic. Recent Advances in Hematology, 3, 238. https://doi.org/10.5005/jp/books/11240_20 
[34] Pang, S.J. and Lazarus, A.H. (2010) Mechanisms of Platelet Recovery in ITP Associated with Therapy. Annals of Hematology, 89, 31-35.

https://doi.org/10.1007/s00277-010-0916-2

[35] Bhoria, P., Sharma, S., Varma, N., Malhotra, P., Varma, S. and Luthra-Guptasarma, M. (2015) Effect of Steroids on the Activation Status of Platelets in Patients with Immune Thrombocytopenia (ITP). Platelets, 26, 119-126. https://doi.org/10.3109/09537104.2014.888546

[36] Liu, W.J., Bai, J., Guo, Q.L., Huang, Z., Yang, H. and Bai, Y.Q. (2016) Role of Platelet Function and Platelet Membrane Glycoproteins in Children with Primary Immune Thrombocytopenia. Molecular Medicine Reports, 14, 2052-2060. https://doi.org/10.3892/mmr.2016.5504

[37] Fan, L. and Wei, W. (2014) Platelet Parameters in Children with Idiopathic Thrombocytopenic Purpura Clinical Application Analysis. Clinical and Experimental Medicine, 13, 124-126.

[38] Kim, J.T., Lee, K.W., Kim, S.N. and Kim, M.K. (2002) Changes in Mean Platelet Volume and Platelet Distribution Width after Therapy in Childhood Idiopathic Thrombocytopenic Purpura. Korean Journal of Pediatrics, 45, 505-511.

[39] Noris, P., Klersy, C., Gresele, P., Giona, F., Giordano, P., Minuz, P., Loffredo, G., Pecci, A., Melazzini, F., Civaschi, E. and Mezzasoma, A. (2013) Platelet Size for Distinguishing between Inherited Thrombocytopenias and Immune Thrombocytopenia: A Multicentric, Real Life Study. British Journal of Haematology, 162, 112-119. https://doi.org/10.1111/bjh.12349 